\title{
Analysis of synchronous interpolated signals in Tactical Sensor Network-Visible light communication (TSN-VLC) link
}

\author{
Atul Ranjan Srivastava ${ }^{a}$, Amit Kumar Sharma $^{b}$, Gaurav Sharma $^{c}$, Neha Sharma $^{d}$ \\ Asst. Prof. SRMSCET, Bareilly, India ${ }^{\mathrm{a}-\mathrm{c}}$, Asst. Prof., Bareilly, India ${ }^{\mathrm{d}}$ \\ 1604atul@gmail.coma , amitpiyoosh@gmail.com ${ }^{\mathrm{b}}$, gaurav.06ec@gmail.com ${ }^{\mathrm{c}}$, nehasharma.jis@gmail.com ${ }^{\mathrm{d}}$
}

\begin{abstract}
Sampling frequency offset (SFO) is an important issue in the tactical sensor network (TSN) based visible light communication (VLC) systems in Tactical Sensor Network. A digital interpolation can be used to effectively compensate the SFO. In such case, oversampling at the receiver ADC is required to mitigate the aliasing effect due to imperfect DACs and nonlinearity of visible light sources that cause extra frequency components inside/outside the signal spectrum. The oversampling factor is mainly determined by the order of the digital interpolation filter and TSN links. The design of the TSN-VLC receiver incorporating the digital interpolation filter is vital as it affects not only the transmission performance but also the complexity of signal processing. To evaluate the feasibility of the digital interpolation-based SFO compensation schemes for cost-sensitive TSN applications. Based on the real-time VLC tactical sensor network, the comparison in the VLC-TSN transmission performance and DSP complexity between different interpolation-based SFO compensation schemes is analyzed.
\end{abstract}

Key words: Communication, Interpolation, Tactical sensor network (TSN), Time Stretching, SFO, VLC

\section{Introduction}

To design a practical VLC receiver that mitigates the SFO effect based on the aforementioned principle, a block diagram of the DSP in the OFDM-VLC receiver with the OFDM synchronization is given in figure1. The digitized signal after the ADC is recovered by the DSP functions including synchronization, SFO estimation/compensation, fast Fourier transform (FFT), equalization and quadrature amplitude modulation (QAM) demapping. The synchronization function is applied to detect the start of an OFDM frame/symbol. Error vector magnitude (EVM) is calculated for quantifying the transmission performance. It is noted that as a feedback loop is applied to track the SFO estimated after the FFT, there is a delay of up to two symbol periods (FFT and SFO estimation) in compensating the SFO, which is usually not considered in the simulation or offline signal processing. [1]

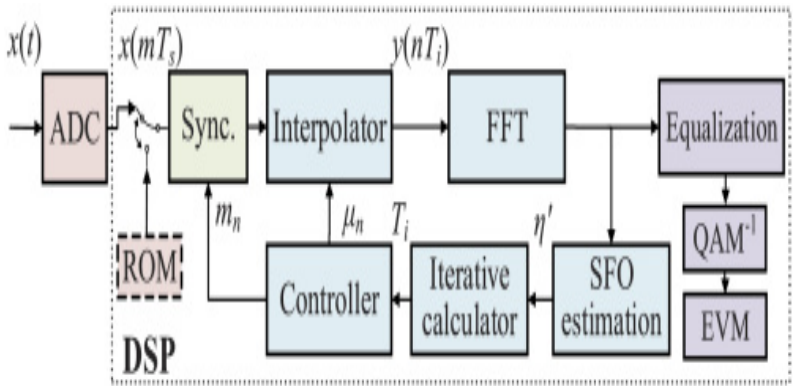

Figure1: DSP in the OFDM-VLC in Tactical Sensor Network

\subsection{Introduce the Problem}

This usually requires a training sequence added before the information data, which is related to the 
design of the frame structure in the spectral density spectrum of tactical sensor network. Also aliasing effect resulted from the SFO and LED nonlinearity can be mitigated by digitally resampling the received synchronous interpolated signal with a Farrow-structured interpolator.[2] It is noted that the digital interpolator is applied to compensate the SFO instead of the LED nonlinearity in a nonlinear VLC link in TSN as shown in figure2.

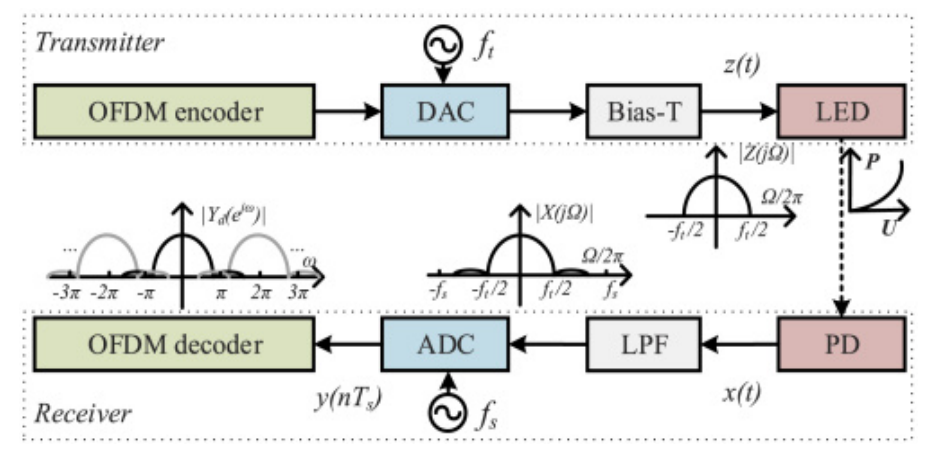

Figure2: Synchronous Interpolator

\subsection{Sub topic}

If we talk about the related structure of this proposed tactical sensor network there were number of dimensions related VLC link. The outline proposed firstly survey communication protocols for the five layers in TCP-IP models. The upcoming step is special constraints of sensor network. The time domain communication strategies are very dominating mechanism in sensing environment. Therefore, it is essential to establish communication topology in VLC link. [3-6]The proposed survey model is as shown by figure3.

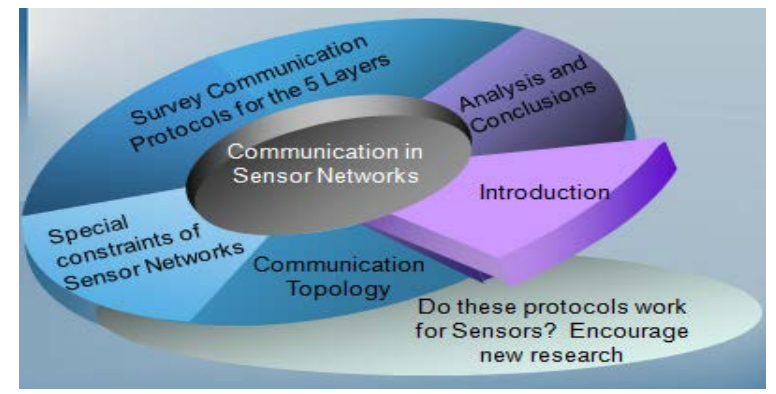

Figure3: Survey outline

The electromagnetic interference and hazard related with visible light communication is completely nil. Unlike RF technology, visible light communication technology has line of sight. Radio Frequency technology has poor security, while Visible light communication technology has very high security. Unlike RF, VLC can be used for dual purposes- for both illumination and communication. The noise sources, which could interfere with the VLC, include sunlight and other ambient lights. This can be easily solved by using this technology in closed areas. Loss of data or data leakage is a serious threat to the national security. Defense and security services use Intranet (private network) services for data storage and data transfer. One of the main problems faced by defense and security is the data breaching or data leakage. Many forms of communication are being utilized within the organization, such as Instant Messaging; VOIP; etc., beyond traditional email, more chances for data leakage have emerged. [7]Every year many confidential data are being leaked or spilled. Data might get hacked or 
exploited while confidential data are being transferred from one system to another. If we are able to implement visible light communication technology in defense and security, then the smallest pilferage and sabotage of data during the confidential data transfer can be avoided. Immense ratio of the detector size and signal wavelength. The other link configuration of this type of classification is the hybrid design method in which the transmitter and receiver can have different levels of directionalities, such as a narrow beam transmitter directed to a specific point and a wide FOV receiver, which are not aligned to a particular direction. The second type of design choice is the existence of a LOS path between the transmitter-receiver pair. There are two options in this category of configuration. First is the Line of Sight (LOS) configuration in which no interruption or obstacles are present between the transmitter and receiver. Thus, no reflection consideration is considered that simples the path loss calculation. Besides, high power efficiency is achievable. In contrast, in the Non-LOS architectures, signals emerge from the source do not directly arrive at the receiver.[8-10] They are reflected from different surfaces or objects and arrive in different time intervals to the receiver. This causes multipath distortions and makes the estimation of path loss much more difficult. The Non-LOS architecture with no directed transmitter-receiver pair is called diffuse systems which is the most robust system and easy to implement for especially the mobile communication scenarios. [11]

\section{Review}

All simulations were designed and run in a custom simulator built in MATLAB. The simulator is designed to simulate any number of sensor nodes in either a uniform spacing or a random distribution. This flexibility allows the testing of the effectiveness of the modified Loading protocol in the scenarios of deliberate or random placement of nodes.
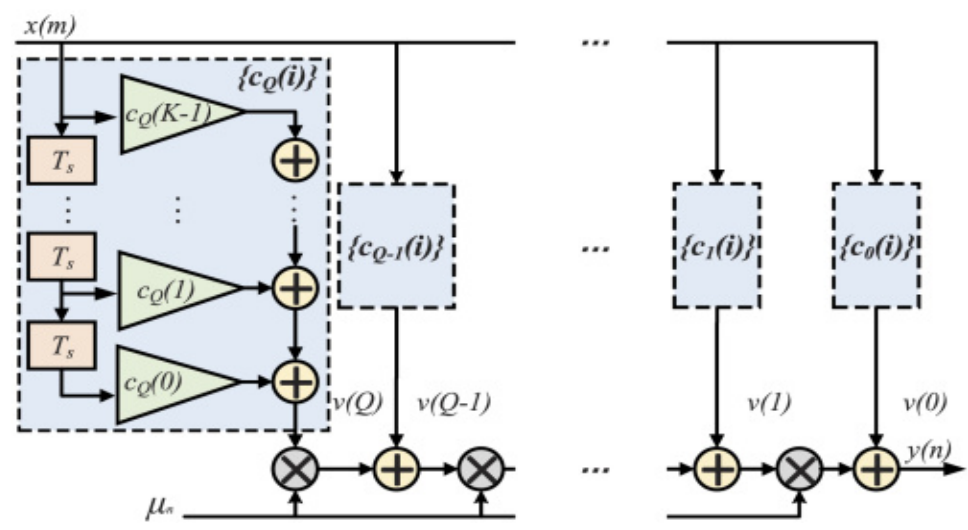

Figure4: Analysis of synchronous interpolated TSN

Tactical Sensor Networks (TSNs) consist of small nodes with sensing, computation, and wireless communications capabilities. Many routing, power management, and data dissemination protocols have been specifically designed for WSNs where energy awareness is an essential design issue. The focus, however, has been given to the routing protocols which might differ depending on the application and network architecture. Due to recent technological advances, the manufacturing of small and low cost sensors became technically and economically feasible. The sensing electronics 
measure ambient conditions related to the environment surrounding the sensor and transform them into an electric signal. Processing such a signal reveals some properties about objects located and events happening in the vicinity of the sensor. A large number of these disposable sensors can be networked in many applications that require unattended operations. A Tactical Sensor Network (TSN) contains hundreds or thousands of these sensor nodes.

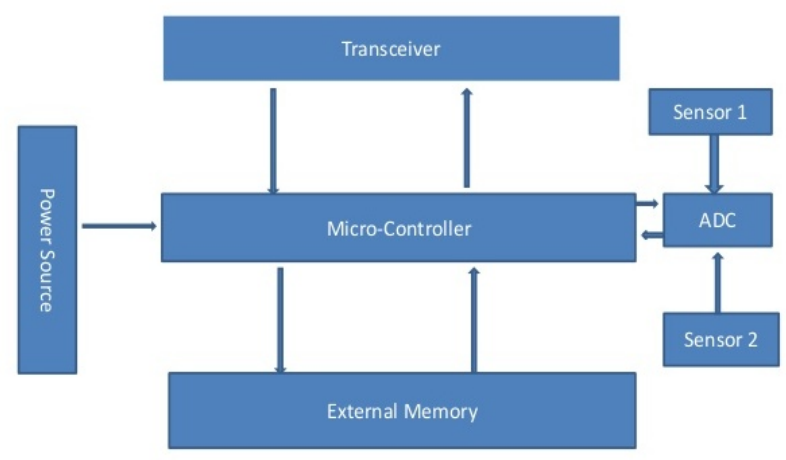

Figure5: Architecture of tactical sensor network

Point out our basic architecture of tactical sensor network having definite sensor node. There are c ertain blocks which involve transceiver which have the functionality to communicate with several 1 inks including VLC.The heart of the architecture is a microcontroller which have memory to perfo rm the required operation during link establishment procedure in tactical sensor network visible lig ht communication system. [12-14] To enable this architecture there exist a power supply. Also a a nalog to digital converter is present to convert analog signal into digital signal in between the diff erent sensor nodes. The sensor nodes are configured to construct sensor network mechanism tactica lly.

\section{Proposed Analysis}

If the broadcasts are captured for a listener and played back, merely slowing down the playback of the audio is an easy solution for this fast speed. Sadly, this comes at the expense of seriously distorting the sound's pitch content. There is a much darker sound in the slow down playback, while the speed up version has a much higher sounding pitch. An instance of this is if you were to put your finger on a record to slow down the speed of play and note that the pitch is much lower.
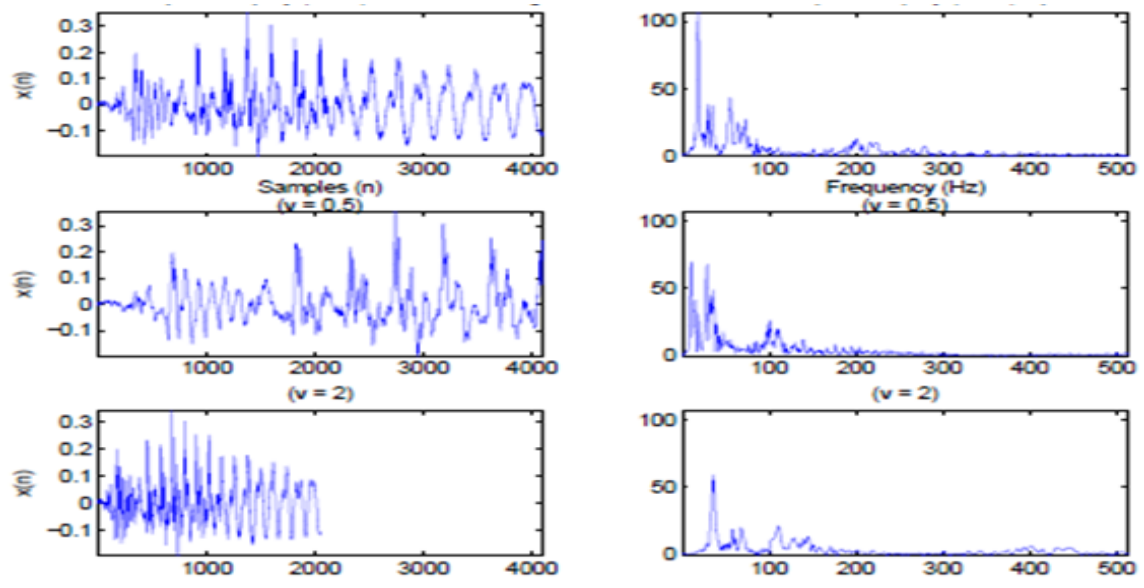

Figure 6. Spectrum of synchronous interpolated signal in TSN-VLC system 
Figure 6 illustrates this using a sound segment that is shortened and elongated to show the change in the spectral information. On the left side of the figure the time information is displayed. Where, with the same sample range, the information is either compressed or elongated. Owing to the compression and extension of the original signal, the frequency information or range has also modified. Cross-correlation is a way of evaluating over a time-lag the similarity of two waveforms. In signal processing, it is used extensively to find smaller wave forms in a longer sample, which contributes to pattern recognition. This cross-correlation knowledge will be used to find the position with the highest similarity between the time-shifted signal overlap intervals.

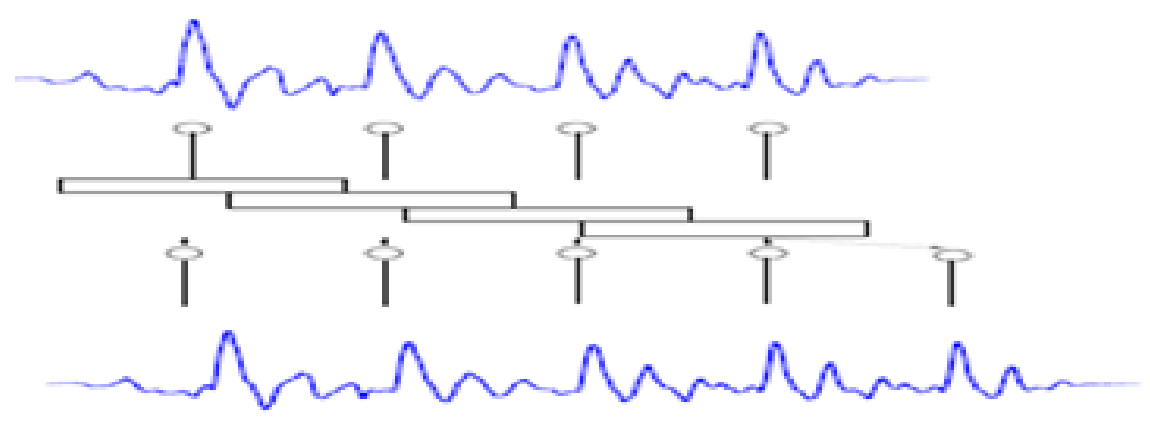

Figure 7: Depiction of the PSOLA algorithm

Pitch-synchronous Overlap Add utilizes the hypothesis that a pitch characterizes the input signal. It takes advantage of the pitch's expertise to synchronize time segments correctly, preventing pitch discontinuities. The PSOLA algorithm is basically divided into two phases: the first phase analyses and extracts the pitch information from the input sound segments and the second phase synthesis a time extended version by overlapping and inserting time segments extracted by the analytical phase as shown in figure 7.

\section{Results}

While we're not going to concentrate on sample rate conversion, when a signal is extended in the time domain, it is necessary to understand the overall impact on the frequency data. The index referring to the highest correlation is now used as a way to overlap the signals. (Figure8).

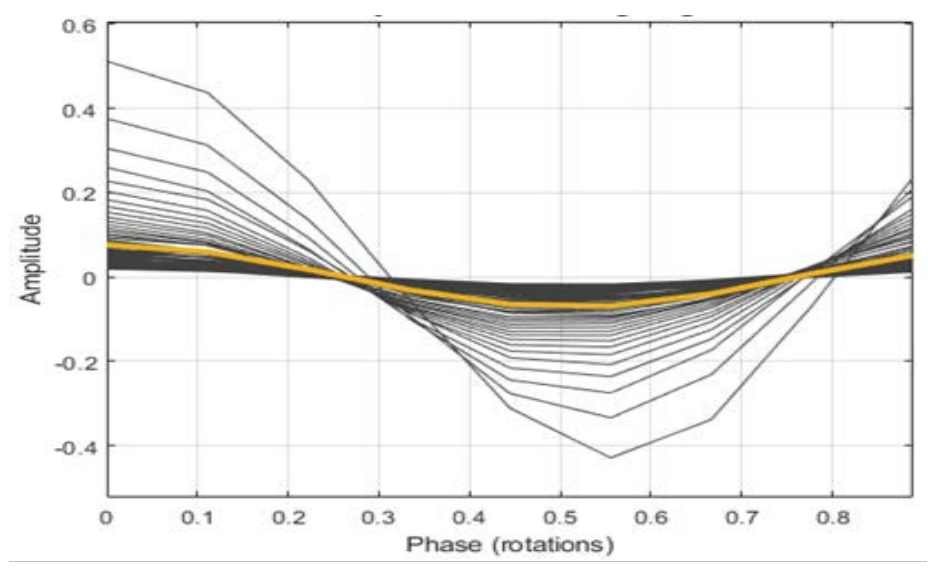

Figure 8: Average of synchronous interpolated signals related o SFO 
For visible light, those frequencies lie roughly between $400 \mathrm{THz}$ and $700 \mathrm{THz}$. That implies oscillation cycles with durations of only a few femto seconds. While some sources emit quasi-monochromatic light, having a well defined optical frequency, others can have a very large optical bandwidth of hundreds of terahertz. (Figure9)

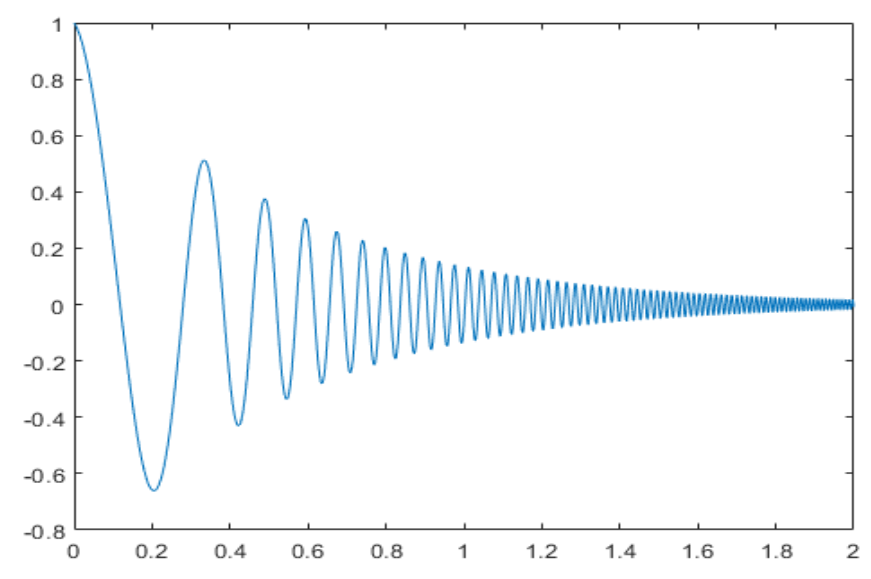

Figure 9 SFO Output of VLC link

As the visible light spectrum is 10,000 times larger than the radio frequency spectrum, VLC is regarded as a solution to RF bandwidth limitations. Industry has generated very high data transmission rates, making it competitive. Though the signal cannot penetrate obstructions such as walls, a direct line of sight is not required as long as long as light is reflected from other surfaces. The LED lighting must be ON for the signal to transmit but can be dimmed to very low levels. VLC has an advantage over Wi-Fi in that transmission does not cause electromagnetic interference. The exact relation between Photocurrent and distance travelled in VLC link is analyses by two domains. First are Incandescent and other fluorescent. (Figure10).

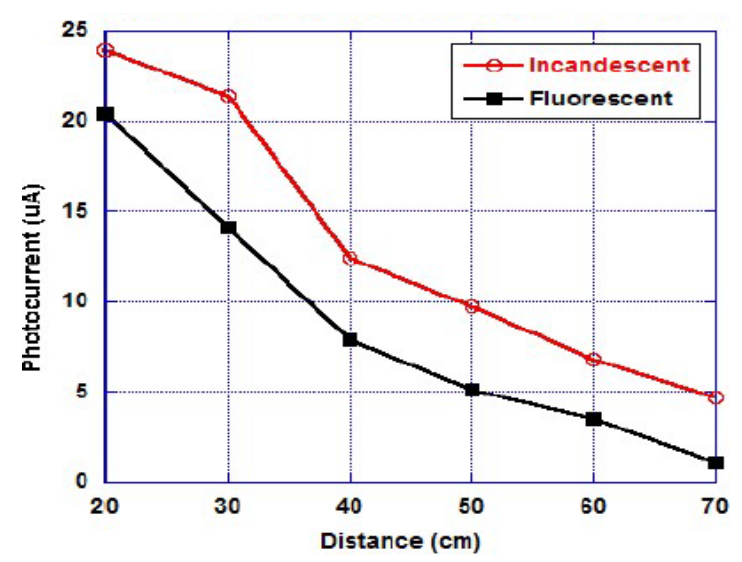

Figure 10 Relation between Photocurrent and distance travelled in TSN-VLC link

Pitch is an attribute which is correlated with a sound's pitch. It is categorized into a certain pitch depending on the frequency of the signal. The use of pitch data will play a crucial role in time stretching, although the two are not identical. The zero-crossing rate defines how many times in a given period the waveform crosses the zero axis. At a measurement interval of 20ms, Figure 11 
displays a $100 \mathrm{~Hz}$ sine wave. In the entire sample frame, there are four zero-crossing in quantum key distribution (QKD). (Figure11).

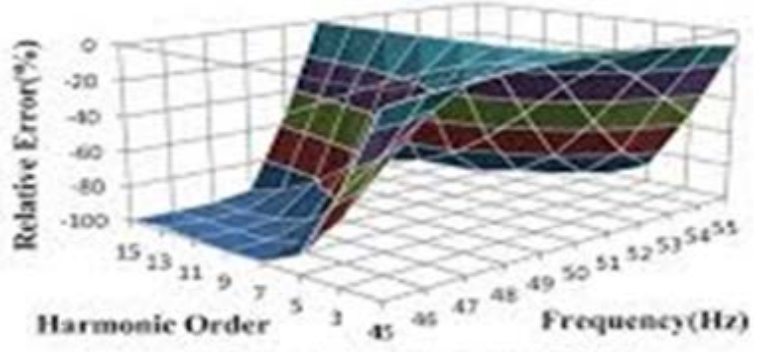

Figure11 TSN-VLC Zero crossing for a fs= $100 \mathrm{~Hz}$ in QKD

\section{Discussion}

We have used documented signals for the previous implementations of time-stretching algorithms where the statistics were known during the entire processing cycle. Hopefully, this Spread Spectrum was instructive and acquired all the necessary tools to design, simulate, display, and analyze results in the various forms explored; time plots, SFO, spectra plots, frequency domain graphs, and PSOLA. This information should equip us to build systems that are more complex. It was identified that a direct correlation exists between the SNR and the quality of the connection associated with a given link between nodes. Knowledge can be readily obtained from software tools for network management. The analysis is based on the error rate and the secure communication rate as functions of distance for three quantum-key-distribution (QKD) protocols. We consider the secure communication rate of the DPSK protocol to analyze it. In this study, we have found the usage of the TSN in diverse fields. Due to the rapid and progressive development of society, continuous innovation is an utmost requirement for human civilization and its development. The scope of TSN-VLC system applications is not limited to, environmental monitoring but also the surveillance of criminal activities, healthcare, and infrastructure monitoring. Each application has its own features and requirements.

\section{References}

1. I. F. Akyildiz, W. Su, Y. Sankarasubramaniam, E. Cyirci, “ A survey on Sensor Networks”, Computer Networks, 38(4):393-422, March 2002

2. Chee-Yong Chong, S. P. Kumar, "Sensor networks: evolution, opportunities, and challenges”, Proceedings of IEEE, pp 1247-1256, August 2003

3. Bal, M. Industrial applications of collaborative Wireless Sensor Networks: A survey. In Proceedings of the 2014 IEEE 23rd International Symposium on Industrial Electronics (ISIE), Istanbul, Turkey, 1-4 June 2014. 6. Chee-Yee, C.; Kumar, S. Sensor networks: Evolution, opportunities, and challenges. Proc. IEEE 2003, 91, 1247 1256.

4. R. Ghanadan, K. Guan, S. Mo, and J. Hsu, "Improving Network Reachability and Data Rate in Tactical Wireless Networks via Collaborative Communications” in Communications Workshops, 2008.ICC Workshops '08. IEEE International Conference on 19-23 May 2008pp. 316 - 320 Available: IEEE Xplore, http://ieeexplore.ieee.org Accessed: June 9, 2010.

5. Sidiropoulos, N. D., Davidson, T. N., \& Luo, Z. Q. (2006). Transmit beamforming for physical-layer multicasting. IEEE transactions on signal processing, 54(6), 2239-2251.

6. Barroca, N.; Borges, L.; Velez, F.; Monteiro, F.; Górski, M.; Castro-Gomes, J. Wireless sensor networks for 
temperature and humidity monitoring within concrete structures. Constr. Build. Mater. 2013, 40, 1156-1166

7. J.P.G. Sterbenz, R. Krishnan, R. R. Hain, A. W. Jackson, D. Levin, R. Ramanathan, and J. Zao, "Survivable Mobile Wireless Networks: Issues, Challenges, and Research Directions,” Workshop on Wireless Security Archive Proceedings of the 1st ACM Workshop on Wireless Security Atlanta, GA, USA, 2002, pp.31-40.

8. R. J. Ellison, D. A. Fisher, and R. C. Linger, “An approach to survivable systems,” In: NATO IST Symposium on Protecting Information Systems in the 21st Century, 1999

9. Gkikopouli, A.; Nikolakopoulos, G.; Manesis, S. A survey on Underwater Wireless Sensor Networks and applications. In Proceedings of the 2012 20th Mediterranean Conference on Control \& Automation (MED), Barcelona, Spain, 3-6 July 2012

10. A. Fujimura, S. Y. Oh, and M. Gerla. Network coding vs. erasure coding:Reliable multicast in ad hoc networks. In In Proceedings of IEEE Military Communications Conference 2008(Milcom 08), November 2008.

11. Trellis Ware Technologies, Inc. (2011). Web services API documentation. San Diego: TrellisWare Technology, Inc.

12. Pahuja, R.; Verma, H.; Uddin, M. A Wireless Sensor Network for Greenhouse Climate Control. IEEE Pervasive Comput. 2013, 12, 49-58. CrossRef.

13. Trellis Ware Technologies, Inc. (2012b). MANET mission configuration guide. San Diego: Trellis Ware Technologies, Inc.

14. Zenia, N.; Aseeri, M.; Ahmed, M.; Chowdhury, Z.; Shamim Kaiser, M. Energy-efficiency and reliability in MAC and routing protocols for underwater wireless sensor network: A survey. J. Netw. Comput. Appl. 2016, 71, 72-85. 